\title{
Observation of cosmogenic nuclide Be-7 concentrations in the air at Bangkok and trajectory analysis of global air-mass motion
}

\author{
Soichiro Suzuki ${ }^{* a}$, Hirohisa Sakurai $^{a}$, Fuyuki Tokanai $^{a}$, Emiko Inui $^{b}$, Hirofumi \\ Shimizu $^{c}$, Kimiaki Masuda ${ }^{d}$, Warit Mitthumsiri ${ }^{e}$, David Ruffolo ${ }^{e}$, Ronald \\ Macatangay $^{f}$, Satoshi Kikuchi $^{a}$, Yutaka Kurebayashi ${ }^{a}$ \\ ${ }^{a}$ Department of Physics, Yamagata University \\ ${ }^{b}$ Radioisotope Laboratory, Yamagata University \\ ${ }^{c}$ Department of Earth and Environmental Sciences, Yamagata University \\ ${ }^{d}$ ISEE. Nagoya University \\ ${ }^{e}$ Department of Physics Faculty of Science, Mahidol University, Bangkok, Thailand \\ ${ }^{f}$ National Astronomical Research Institute of Thailand, Chiang Mai, Thailand \\ E-mail: Vamagata.tokanaieknegmail.com \\ sakuraiesci.kj.vamagata-u.ac.jp tokanaidsci.kj.vamagata-u.ac.jp \\ Inuidsci.kj.yamagata-u.ac.jp, s12e014est.yamagata-u.ac.jp \\ kmasudadstelab.nagoya-u.ac.jp, warit.mitamahidel.ac.th \\ david.rufemahidol.ac.th, ronmcdoegmail.com
}

\begin{abstract}
We have been continuously observing the daily Be-7 concentrations at Bangkok in Thailand $\left(13.765^{\circ} \mathrm{N}, 100.526^{\circ} \mathrm{E}\right)$ since June 2014 to investigate the relationship between solar activities and the variation of cosmogenic nuclides in low latitude. The Be-7 concentrations show a clear seasonal variation with the high and low concentrations during January to May and June to December, respectively, for each year. In order to understand the seasonal variations, we calculated back trajectories of air mass by HYSPLIT. The calculation indicates that the back trajectories are distinguished three patterns in a year, which are "Indian Ocean trajectories", "Continental trajectories", and "Pacific Ocean trajectories". Moreover, we estimated monthly amount of Be-7 coming to Bangkok for each trajectories by EXPACS. From these findings, the amount of Be-7 is high during Continental and Pacific Ocean trajectories. On the other hand, one is low during Indian Ocean trajectories. This phase of calculated Be-7 can explain the seasonal variation. Comparing this phase and the phase of observed Be-7 concentrations, there is a time lag of one or two months.
\end{abstract}

35th International Cosmic Ray Conference - ICRC2017

10-20 July, 2017

Bexco, Busan, Korea

${ }^{*}$ Speaker. 


\section{Introduction}

Cosmogenic radioactive nuclide $\mathrm{Be}-7$ of the half-life of 53 days is produced by spallation interactions between cosmic rays and nitrogen or oxygen in the atmosphere. Once produced, they are immediately oxidized and then attach to aerosols. The aerosols with Be-7 fall down to the ground surface by dry deposition and/or wet deposition.

The nuclide Be-7 is a useful tool to investigate time variations in cosmic rays coming to the whole Earth, because the Be-7 is globally produced in the atmosphere and are fallen down to the surface through global scale air-mass transport. Thus, we have been observing daily Be-7 concentrations sampling aerosols in surface air at Bangkok located at a high cut-off rigidity of the geomagnetic field. There are few observations for the area compared with those for the midlatitudes[四].

The Be-7 concentrations in surface air are disturbed by a global scale air-mass transport after the Be-7 is produced in the atmosphere. The transport includes the air-mass exchange between the stratosphere and the troposphere, the vertical transport in the troposphere, and the horizontal transport such as from the polar to the tropical region. Therefore, when investigating the time variations in cosmic rays such as solar modulation from those of Be-7 concentrations, it is important to clarify which region that the Be-7 at a sampling site are coming from which region and through which the three-dimensional paths through the atmosphere.

So, we calculated the Be-7 coming to Bangkok employing two kinds of simulators of the Hybrid Single-Particle Lagrangian Integrated Trajectory model (HYSPLIT) and Excel-based Program for calculating Atmospheric Cosmic-ray Spectrum (EXPACS)[[2][3]. The air-mass trajectories to the sampling site are simulated using the backward function of HYSPLIT and the production rates of Be-7 are simulated for an arbitrary point in the earth atmosphere using EXPACS. In this report, we describe the time profiles of observed Be-7 concentrations at the Bangkok sampling site for three years, the simulated air-mass trajectories, and the amount of Be-7 coming to Bangkok by the simulation, discussing the relationships between them.

\section{Experiment and Method}

\subsection{Sampling and Measurement}

Daily Be-7 concentrations in the atmosphere have been continuously observed at the Faculty of Science, Mahidol University in Bangkok since June, 2014. The sampling location is at $13.765^{\circ}$ $\mathrm{N}, 100.526^{\circ} \mathrm{E}$, and the altitude is $15 \mathrm{~m}$. A high-volume air sampler (HV-500R) manufactured by SIBATA Company is set for collection of aerosols from the air. The air was collected at 500L per minute. The aerosols with Be-7 is trapped in a sheet of the glass fiber filter with the diameter of $110 \mathrm{~mm}$ (ADVENTEC GB-100R). The radioactivity of Be-7 in the filter, which emits $\gamma$-ray of $477.6 \mathrm{keV}$, was measured using the high-purity germanium $\gamma$-rays detector GCW3023 (CANBERRA) at the $20 \mathrm{~m}$ underground laboratory of the Institute for Cosmic Ray Research (ICRR), Tokyo University. The background level of the detector system is $6.0 \times 10^{-3} \mathrm{cpm}$ on the equivalent channel to the $\gamma$-rays energy of the Be-7. 


\subsection{HYSPLIT and EXPACS}

We analyzed the trajectories of air mass motion using HYSPLIT. In this simulation, the meteorological data set GDAS1 (Global Data Assimilation System) was provided by the NOAA's (National Oceanic and Atmospheric Administration) Air Resources Laboratory (ARL). The back trajectories were calculated for all on one dates from June in 2014 to December in 2016 as follows: The end point of trajectories, at which the sampling site, was set at $13.765^{\circ} \mathrm{N}, 100.526^{\circ} \mathrm{E}$, with the altitude of $3 \mathrm{~m}$. The trajectory was backward traced up to 30 days with the time interval of 1 hour. The number of calculated trajectories on one date is 24 paths with the time interval of 1 hour. To calculate the production of Be-7 on the trajectory air-mass boxes with $1 \mathrm{~km}$ cubic cells are put on the trajectory at 1 hour interval. So the number of air-mass boxes reaching the end point is 720 boxes for the trace duration of 30 days

To calculate amount of Be-7 on each of air mass trajectories flown in Bangkok, we investigated using EXPACS simulation program. EXPACS can calculate the energy spectrum of neutrons and protons at an arbitrary altitude, latitude, longitude, and date considering the vertical cut-off rigidity and the solar modulation of the primary galactic cosmic rays spectra. Using EXPACS, Be-7 production rate $P(x, y, z, t)$ [atoms/g/s] in a time $t$ and three-dimensional coordinates $(x, y, z)$ is calculated as follows:

$$
P(x, y, z, t)=\int_{E_{1}}^{E_{2}} F_{n, p}(E, x, y, z, t) N_{N, O}(x, y, z) \sigma_{B e-7}(E) d E,
$$

,where $F_{n, p}(E, x, y, z, t)$ [particles $/ \mathrm{cm}^{2} / \mathrm{s}$ ] is the energy spectrum of neutrons or protons, $N_{N, O}(x, y, z)$ [atoms $\left./ g_{\text {air }}\right]$ is a number of nitrogen or oxygen in air mass, and $\sigma_{B e-7}\left[\mathrm{~cm}^{2}\right]$ is the cross-section of Be-7 production. $E_{1}$ and $E_{2}$ are $10[\mathrm{MeV}]$ and $100[\mathrm{GeV}]$, respectively.

The Be-7 in the troposphere, in general, are shown by a differential equation in the box model as follows:

$$
\frac{d N_{t}}{d t}=P-\lambda N_{t}+k_{s} N_{s}-k_{t} N_{t}
$$

, where $N_{t}, N_{s}$, and $P(x, y, z, t)$ are Be-7 at the troposphere and stratosphere, and production rate of Be-7, respectively, and $k_{t}, k_{s}[$ [⿴囗十丁][可], and $\lambda$ are removal rates from boxes in the troposphere and the stratosphere, and disintegration constant of Be-7, respectively.

Since we focused on the air mass trajectories in the troposphere as the first attempt, in the calculation the exchange between the troposphere and the stratosphere was neglected and hence $k_{s}=0$. Also, assuming equilibrium between the production rate and the remove rate of $\mathrm{Be}-7$ in the cell, the amount of Be-7 in each cell on the trajectory was calculated. Moreover, as the Be-7 disintegrated in and was removed out of the cell until the cell reaching the end point, the effect was taken account for the amount of Be-7 as follows:

$$
N(\tau)=\frac{P(x, y, z, t)}{\lambda+k_{t}} e^{-\left(\lambda+k_{t}\right) \tau},
$$

, where $\tau$ is the trace back time. Finally, for a trajectory traced back with the maximum trace back time $\tau_{\max }[$ [6], the total amount of Be-7 reaching the end point was shown as follows:

$$
N\left(\tau_{\max }\right)=\int_{\tau=0}^{\tau=\tau_{\max }} N(\tau) d \tau
$$


To compare with the monthly profile of observed Be-7 concentrations, the total amount of Be-7 was calculated for the trajectories with the time resolution of 1 hour for one month and then they were averaged.

\section{Results and Discussion}

\subsection{Time profiles of the Be-7 concentrations and trajectories by HYSPLIT analysis}
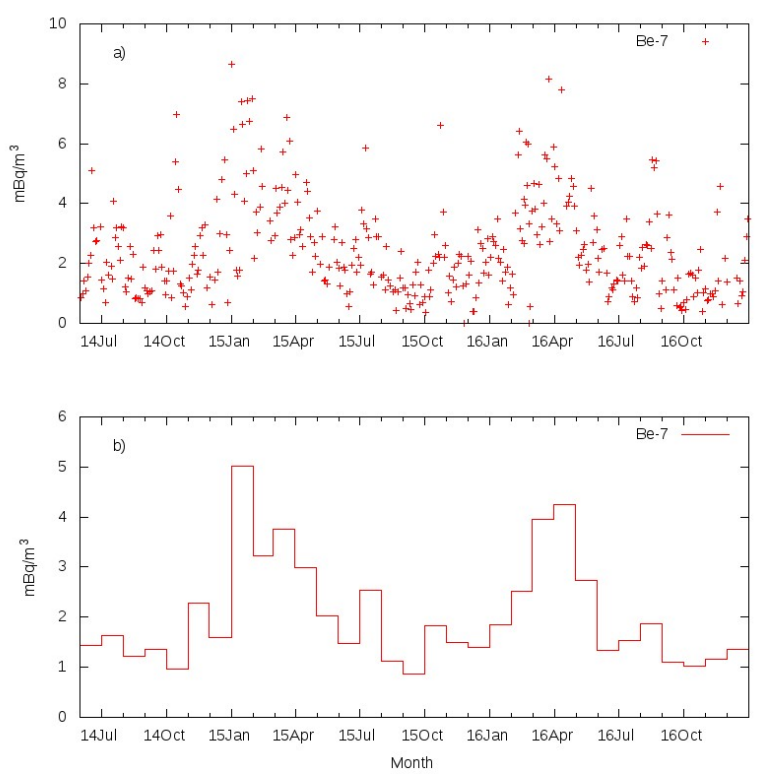

Figure 1: Time profiles of daily and monthly Be-7 concentrations in Bangkok

Fig. $\mathrm{Wa}$ ) and b) show the time profiles of the daily and monthly of observed Be-7 concentration from June in 2014 to December in 2016. The Be-7 concentration shows a clear seasonal variation with the high and low concentrations of $3.4 \mathrm{mBq} / \mathrm{m}^{3}$ and $1.5 \mathrm{mBq} / \mathrm{m}^{3}$ during January to May and June to December, respectively, for both years. The peak of high Be-7 concentration is 2.3 times as large as that of the low Be-7 concentration. Fig. $\square$ shows the time profile of Be-7 concentration

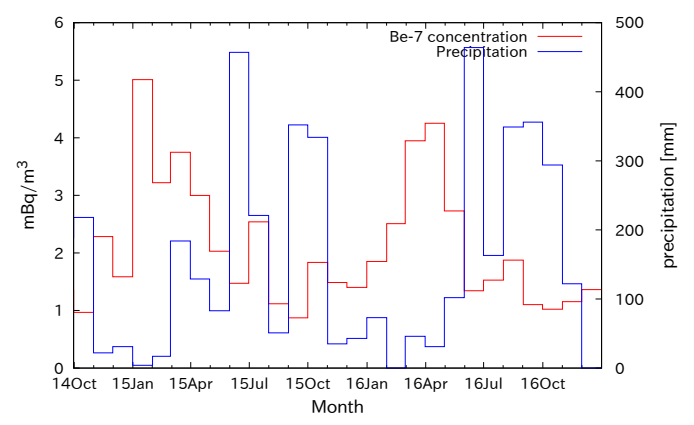

Figure 2: Time profiles of monthly Be-7 concentration and precipitation at Bangkok

compared with that of the monthly precipitation[ $[\mathbb{}]$ in Bangkok from June in 2014 to December 

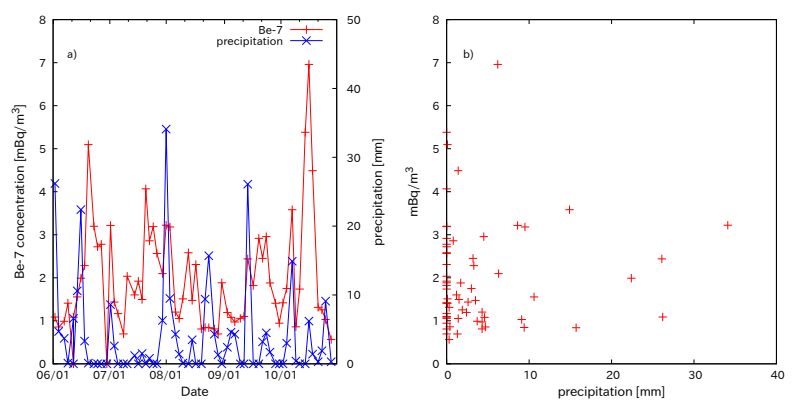

Figure 3: a) Time profiles of daily Be-7 concentration and precipitation during the rain season in 2014 at Bangkok. b) scatter plot between them
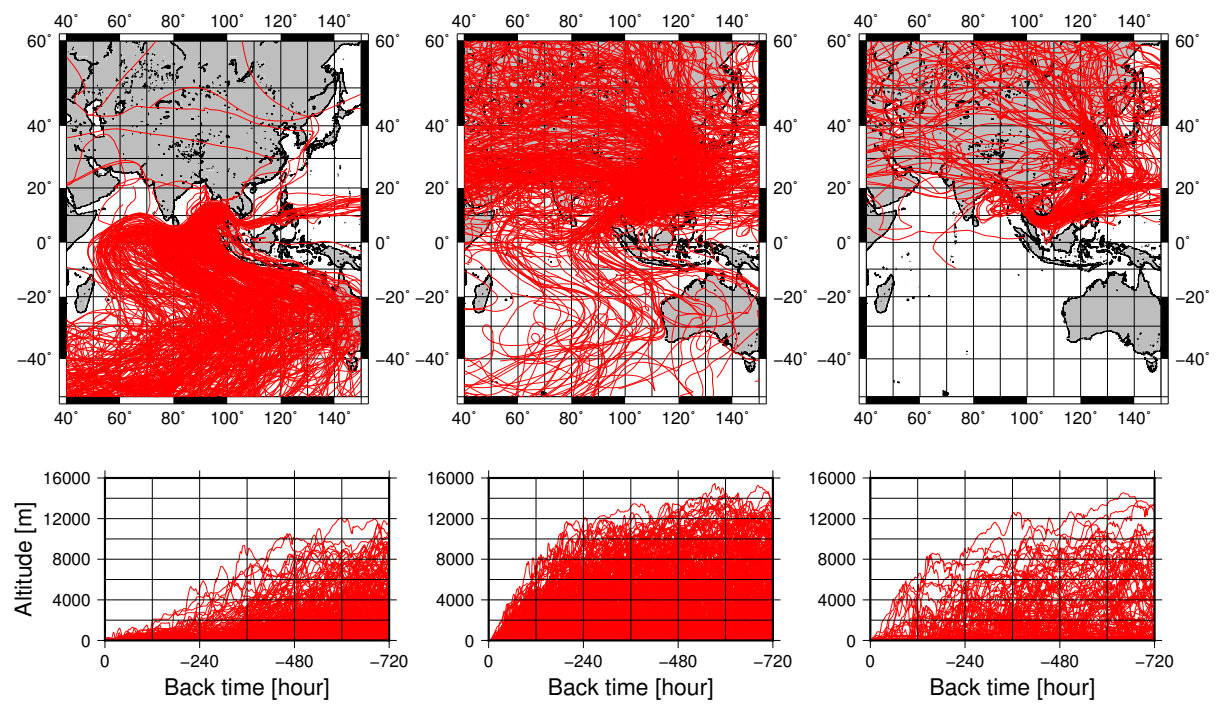

Figure 4: Back trajectories and altitudes of those trajectories. The left panels are from May to September. The center panels are from October to February. The right panels are from March to April.

in 2016. At Bangkok, the rain season is during May to September and the period of small precipitation is during October and April in a year. The seasons correspond to the Indian trajectory, the Continental and Pacific Ocean trajectories of air-mass motion, respectively, as shown in next section. In general, as aerosols are scavenged by rain, it is considered that the Be- 7 concentrations are in low level at rainy season. However, as shown in Fig.(Bla) and b), the relationship between the Be-7 concentration and the precipitation is not so simple from the time profile of daily $\mathrm{Be}-7$ concentration compared with that of the daily precipitation, and the scatter diagram between them for the rain season from June to October in 2014. Therefore, in addition to the local meteorological effects it is necessary to investigate transport processes of Be-7 with global scale.

Fig.⿴囗十 shows the back trajectories coming to Bangkok and the altitudes of trajectories simulated by HYSPLIT. Their trajectories have three patterns, which we call "Indian Ocean trajectory", "Continental trajectory", and "Pacific Ocean trajectory", corresponding to three durations in the year. The Indian Ocean trajectories, the Continental trajectories, and the Pacific Ocean trajectories are coming to Bangkok through the Indian Ocean, the Eurasian Continents, and the Pacific Ocean. Fig.[ [ shows the average trajectories for each month. It is clear that the air masses are 


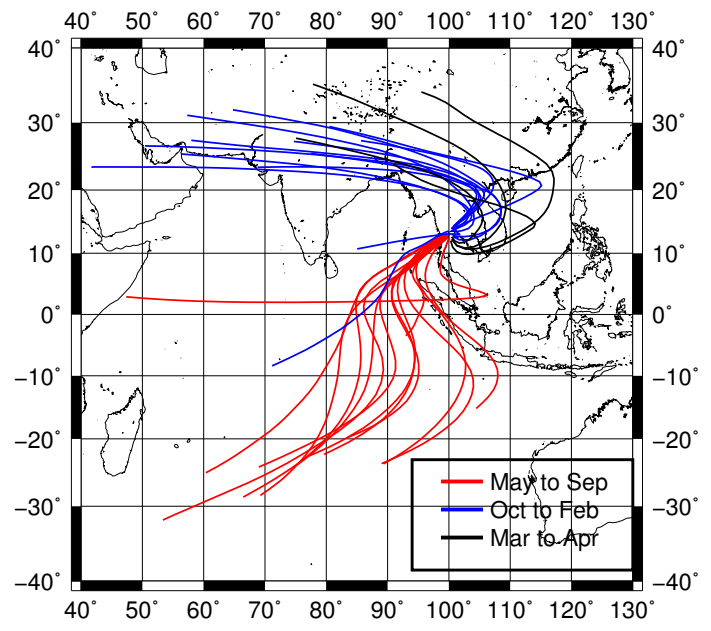

Figure 5: Monthly average latitude and longitude

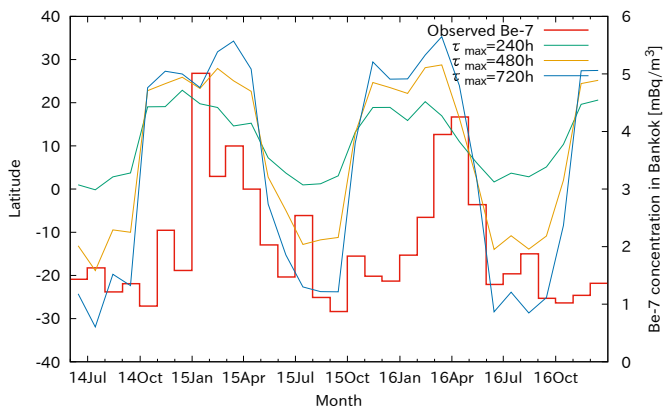

Figure 6: Time profiles of monthly latitudes and the observed Be-7 concentrations

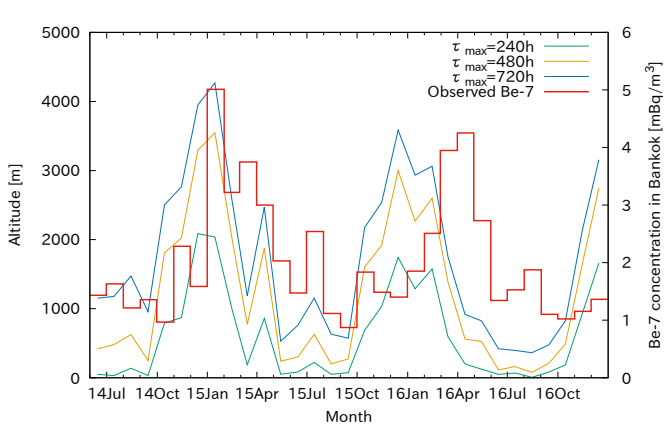

Figure 7: Time profiles of monthly altitudes and the observed Be-7 concentrations

passing through the Indian Ocean, the Eurasian Continent, and the Pacific Ocean, respectively, corresponding to the three seasons.

Fig.6 shows the time profiles of average latitudes for the calculated trajectories for three values $\tau_{\max }(240,480$ and 720 hours), compared with the time profile of the observed Be- 7 concentration. The trajectories during October to April, which are the Continental trajectory and the Pacific Ocean trajectory, are passing through relatively high latitudes of approximately 30 degrees. Also, the trajectories during the May to the September, which are the Indian Ocean trajectory, are passing through roughly tropical latitudes. The periods of the enhanced Be-7 concentrations are included in the duration of higher latitudes.

Fig. $\square$ shows the time profiles of average altitudes for the calculated trajectories for $\tau_{\max }$ (240, 480 and 720 hours), compared with the time profile of the observed Be-7 concentration. The trajectories during October to February, which are corresponding to the Continental trajectory, are passing through relatively higher altitudes. Also, the trajectories during the May to the September, which are corresponding to the Indian Ocean trajectory, are passing through low altitudes. The period of the enhanced Be-7 concentration is shifted from the peak month of the altitudes. Thus, although there are some connection between the seasonal variation of the observed Be- 7 concentrations and the air-mass trajectories, the relationship between them is not so clear. Hence, it is 
important to simulate the amount of Be-7 transported to Bangkok by each trajectory.

\subsection{Simulated Be-7 by EXPACS for trajectories by HYSPLIT}

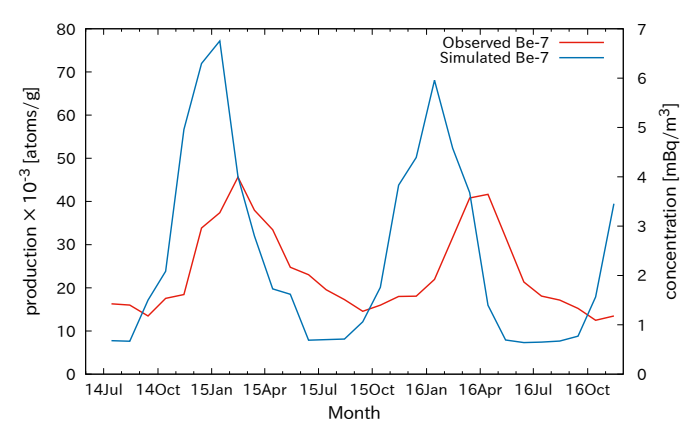

Figure 8: Time profiles of the simulated Be-7 $\tau_{\max }=240$ hours and the observed Be-7 concentrations by moving averages

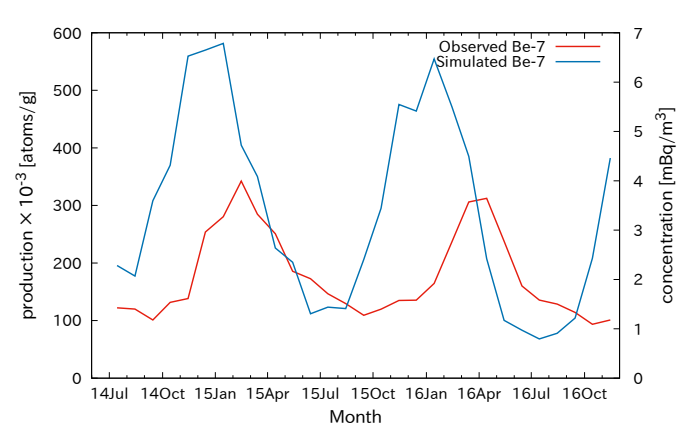

Figure 9: Time profiles of the simulated $\mathrm{Be}-7$ $\tau_{\text {max }}=720$ hours and the observed Be-7 concentrations by moving averages

Fig. 8 shows the monthly time profile of the simulated amount of Be-7 coming to Bangkok and that of the observed Be-7 concentration with the blue and red lines, respectively. Both lines are moving averages over three months. The simulated Be-7 has an enhanced portion during October to April with a peak in January. Also, the period between the two peaks in 2014 and 2015 is just 1 year, indicating an exactly seasonal variation. Although the observed Be-7 concentration has a time profile similar to the simulated Be-7, the peaks are in February with a time lag of approximately

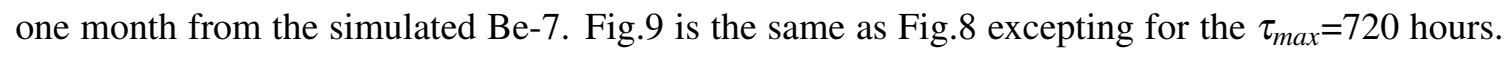
Although the enhancement of the simulated Be-7 shows a broader shape for the larger $\tau_{\text {max }}$, the relationship of the time profiles between the observed Be-7 concentrations and the simulated Be-7 is as good as that for $\tau_{\max }=240$ hours. The time lag might imply a contribution of the stratosphere component of Be-7 because air mass for the Continental and the Pacific Ocean trajectories passes through high altitudes in the troposphere as shown in Fig.t.

Table 1: Yearly Be-7 concentration at Bangkok, Yamagata, Sun spots Number(SSN), neutron intensity in Thule, N(720) simulated Be-7 for $\tau_{\max }=720$ hours in 2014 (from June 2014 to May 2015) and 2015 (from June 2015 to May 2016)

\begin{tabular}{crrrrr} 
Period & $\begin{array}{c}\text { Be-7 at Bangkok } \\
\left(\mathrm{mBq} / \mathrm{m}^{3}\right)\end{array}$ & $\begin{array}{c}\text { Be-7 at Yamagata } \\
\left(\mathrm{mBq} / \mathrm{m}^{3}\right)\end{array}$ & SSN & $\begin{array}{c}\text { Thule NM } \\
(\mathrm{cnts} / \mathrm{hr})\end{array}$ & N(720) \\
\hline \hline 2014 & 2.29 & 4.9 & 94.4 & 4222 & 247.1 \\
\hline \hline 2015 & 2.11 & 5.1 & 57.4 & 4298 & 212.5 \\
\hline \hline $\begin{array}{c}\text { Rate of variability } \\
\text { to 2014(\%) }\end{array}$ & -7.9 & 4.1 & -39.1 & 1.8 & -14.0 \\
\hline \hline
\end{tabular}

Tab.W shows the Be-7 concentration. The neutron intensity in Thule, and the Sunspot number (SSN) for each year from June 2014 and 2015. The neutron at Thule neutron monitor showing modulation of cosmic rays is increasing with $1.8 \%$, corresponding to the SSN of $39.2 \%$ which 
indicates a decrease phase of solar activity. Although the Be-7 concentration at Yamagata, Japan which locates in mid-latitude $\left(38.25^{\circ} \mathrm{N}, 140.25^{\circ} \mathrm{E}\right)$ is increasing with $4.1 \%$ as well as the neutron, the Be-7 concentration at Bangkok is decreasing with $7.9 \%$. Since the simulated Be-7 is decreasing with $14.0 \%$ as well as the observed Be-7 concentration at Bangkok, the decrease against to the increase of neutron at polar region might imply a time lag of produced Be-7 coming to the low latitudes.

\section{Conclusion}

To investigate the relationship between cosmogenic nuclides at low latitude and solar activity, we have been observing the daily Be-7 concentrations at Bangkok, Thailand. The Be- 7 concentration was high $\left(3.4 \mathrm{mBq} / \mathrm{m}^{3}\right)$ during January to May and low $\left(1.5 \mathrm{mBq} / \mathrm{m}^{3}\right)$ during June to December. From the HYSPLIT analysis, the air mass trajectories for three seasons are characterized by three patterns, "Indian Ocean trajectory", "Continental trajectory", and "Pacific Ocean trajectory". So, we calculated the amount of Be-7 for each trajectory using EXPACS simulations. The time profile of the simulated Be-7 is similar to the observed Be-7 concentration excepting for time lag of one month for the peaks between the simulation and observation. We note that scavenging of aerosols by precipitation may also play a role.

\section{Acknowledgment}

The authors also are deeply grateful to the ICRR, which provided the use of the low-background facility for measuring low-level radioactivity. Part of this work was supported by JSPS KAKENHI Grant number 25287051 and JP15K05097. Also, this work is supported partially supported by grant RTA5980003 from the Thailand Research Fund. And this research project is partially supported by Thailand Research Fund awards RTA5980003 and TRG5880173.

\section{References}

[1] S. Kikuchi, H. Sakurai, S. Gunji, F. Tokanai. Temporal Variation of Be-7 concentrations in atmosphere for 8 y from 2000 at Yamagata, Japan: solar influence on the Be-7 time series J. of Environmental Radioactivity, 100,pp.515-521,2009.

[2] T.Sato and K.Niita. Analytical functions to predict cosmic-ray neutron spectra in the atmosphere. Radiat.Res., Vol.166,pp.544-555,2006.

[3] T.Sato, H.Yasuda, K.Nitta, A.Endo, and L.Sihver. Development of parma: Phits based analytical radiation model in the atmosphere Radiat. Res., Vol.170,pp.244-259,2008.

[4] H. Nagai, W. Tada, and T. Kobayashi. Production rates of ${ }^{7}$ Be and ${ }^{10}$ Be in the atmosphere. Nuclear Instruments and Methods in Physics Research B, Vol. 172, pp. 796-801, 2000.

[5] J.F.Bleichrodt. Mean tropospheric residence time of cosmic-ray-produced beryllium 7 at north temperate latitudes. J.Geophys.Res, Vol. 83, pp.3058-3062, 1978.

[6] K.Hirose,M.Aoyama,Y.Katsuragi, and Y.Sugimura. Annual deposition of Sr-90, Cs-137 and Pu-239,240 from the 1961-1980 nuclear explosions: a simple model. Journal of the Meteorological Society of Japan, Vol.65,No.2,pp. 259-277,1987.

[7] http://www.data.jma.go.jp/gmd/cpd/monitor/index.html. Meteorological Agency, Japan. 